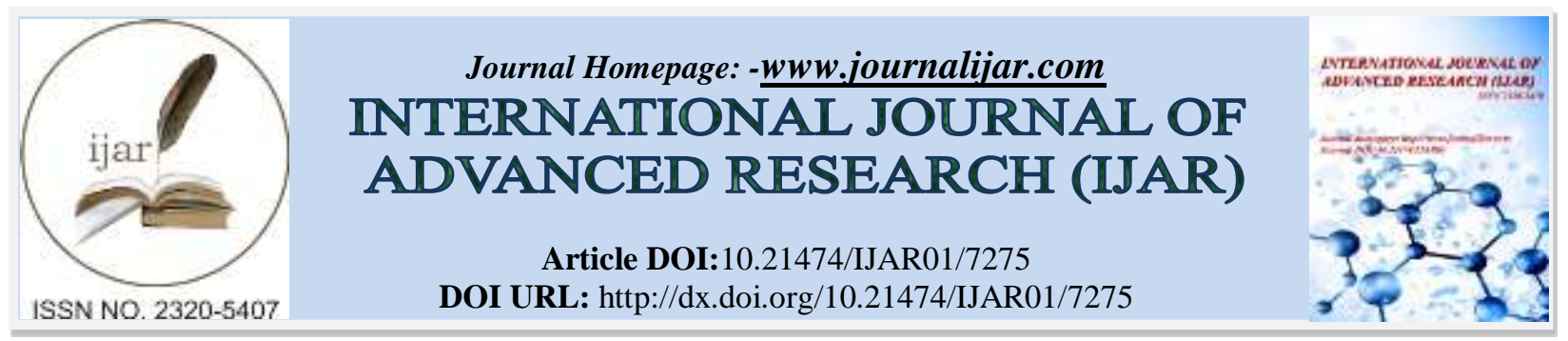

RESEARCH ARTICLE

\title{
SLEEP DISTURBANCES IN MULTIPLE SCLEROSIS PATIENTS: CLINICAL AND POLYSOMNOGRAPHIC STUDY.
}

\author{
Mohamed Tolba El-Sayed Mohamed, MSc; Ahmed Gamal Azab, MD; Osama Abou El-Magd El-Khouli, MD \\ and Tamer Mohamed Ebrahim Belal, MD.
}

\section{Manuscript Info}

Manuscript History

Received: 13 April 2018

Final Accepted: 15 May 2018

Published: June 2018

Keywords:-

Multiple sclerosis, sleep disturbances, polysomnography, clinical.

\section{Abstract}

Background: Sleep disorders and factors contributing to poor sleep in multiple sclerosis (MS) patients remain unclear and most of previous studies used subjective scales for sleep assessment.

Objectives: We aimed to assess the subjective and objective parameters of sleep by evaluating 8-hour polysomnography in MS patients.

Methods: We included 50 MS patients with either relapsing remittent MS (RRMS) or secondary progressive MS (SPMS) and 25 age-and sex-matched controls not diagnosed with sleep disorders. Demographic and clinical characteristics were collected. All participants completed standardized self-report questionnaires including Pittsburg Sleep Quality Index (PSQI), Epworth Sleepiness Scale (ESS), Fatigue Severity Scale (FSS), Beck Depression Index (BDI), Beck Anxiety Index (BAI) scales and underwent history taking, general, neurological examination and nocturnal polysomnography evaluation.

Results: We found $82 \%$ of MS patients were poor sleepers and $50 \%$ of them met the criteria of excessive daytime sleepiness. BDI and BAI scores were significantly higher in poor than good sleepers patients. Thirty six patients $(72 \%)$ had middle insomnia, $46 \%$ had initial insomnia. Nine patients (18\%) had higher apnea/hypopnea index versus none of controls. Seventeen patients $(34 \%)$ had restless legs syndrome and ten $(20 \%)$ had higher periodic limb movement index versus none of controls. Depression, anxiety, pain, nocturia, fatigue, immunotherapy, high disability and poor quality of life were independent predictors for poor sleep.

Conclusions: Overall, $82 \%$ of MS patients had evidence of one or more sleep disturbances. Our study confirms that MS causes sleep fragmentation in terms of both macro and microstructure.

Copy Right, IJAR, 2018,. All rights reserved.

\section{Introduction:-}

Sleep disturbances in multiple sclerosis (MS) patients have received less attention in research as compared to fatigue and depression and clinically under recognized by most physicians and research has primarily focused on prevalence of sleep disorders and the relationship between sleep and fatigue in MS [1]. More than half of MS patients suffer from chronic sleep disturbances and report poor sleep quality [2]. 
Sleep disorders in MS are multifactorial; they may be caused by the degenerative and inflammatory process of the disease which affects areas and cerebral neurotransmitters which may be essential for normal sleep [3]. Even though disrupted nocturnal rest might be directly related to specific sleep disturbances, there is some evidence in the MS literature, that many other demographic and clinical conditions (presence of comorbidities, MS duration and progression, medication effects, pain, muscle spasms, sexual and/or bladder dysfunction, anxiety and depression) should be considered as possible causes of sleep disorders in MS patients [4].

Identifying factors associated with poor sleep in MS is of key importance because sleep dysfunction can potentially exacerbate other MS symptoms and considered as independent predictors of quality of life [4,5]. Indeed, polysomnographic studies have only been conducted on small and unselected patient groups, however, most of previous studies used only subjective sleep questionnaires. Moreover, conflicting measures of sleep disturbances are seen in different studies of MS patients. Sleep studies in patients with MS that included controls are even rare [6].

The main objectives of our study were to evaluate subjective and objective sleep characteristics, pattern and prevalence of poor sleep in a selected group of MS patients and also, to examine the possible causes of sleep disturbance in MS patients through evaluation of the role of demographic, socioeconomic, co-existing conditions, disease severity, stage of disease progression and clinical variables on sleep quality among MS patients.

\section{Subjects and Methods:-}

This is a cross sectional prospective study approved by the Ethical Committee of faculty of medicine, Mansoura University, conducted on 75 individuals; 50 patients diagnosed as having multiple sclerosis according to the revised McDonald criteria 2010 and 25 healthy volunteers (control persons) who match patient group for age and sex.

The patients were recruited and collected from Neurology Department in Mansoura University Hospital (MUH) through outpatients clinic (OPC) from October 2015 to November 2017. The control group was recruited from the MUH workers and the relatives of the patients. All patients and controls gave their informed consent after a detailed explanation of the investigational nature of the study.

Inclusion criteria of patients in this study included acceptance to participate and share in the study, diagnosis of clinically definite MS including patients diagnosed as having relapsing remittent MS (RRMS) or secondary progressive MS (SPMS) according to the revised McDonald criteria 2010 [7], age of the patient $\geq 18$ years, disease lasting more than two years and Expanded Disability Status Scale (EDSS) [8] score of less than 7. 0.

Exclusion criteria of the present study included age of the patient $<18$ years, EDSS score $\geq 7$, patients with severe degree of cognitive impairment as detected by the Mini-Mental State Examination (MMSE) score < 24 [9], patients with past history of primary sleep disorders prior to onset of MS or with severe chronic medical disorders (otorhinological disorders, uncontrolled diabetes mellitus, severe resistant hypertension, hepatic or renal failure and chest diseases) or history of psychiatric disorders (psychosis, major depression or bipolar disorder) preceding diagnosis of MS that could affect sleep, patients with history of drug addiction, alcohol intake or with current history of drug intake that could affect sleep as hypnotics, antidepressant, antipsychotic or antihistaminic drugs and Body Mass Index (BMI) more than 30.

Controls were healthy gender and age matched subjects to MS patients who had no sleep complaints, no medical disorders, and were not receiving any medication that could affect sleep parameters.

Our participants underwent thorough medical and neurological history taking and examination with stress on clinical MS features, coexisting conditions, current therapy and the use of medication either disease modifying therapy (DMT) or symptomatic treatments. Neurological disability measured by Kurtzke's Expanded Disability Status Scale (EDSS) [8] was also assessed in all MS patients.

Subjective measures also were included; such as Pittsburgh Sleep Quality Index (PSQI) [10] to assess the quality and patterns of sleep during the previous month, Epworth sleepiness scale (ESS) [11] to assess subjective sleepiness, Fatigue severity scale (FSS) [12] to assess levels of fatigue and its effect on daily functioning, Beck 's depression inventory scale (BDI) [13] to assess mood related symptoms, Beck 's anxiety inventory scale (BAI) [14] to measure severity of anxiety during the past month and assess effect of anxiety on sleep of those patients and 
Rapid eye movement Behavior Disorders (RBD) Screening Questionnaire (RBDSQ) [15] to assess sleep behavior. PSQI score > 5 indicates poor sleep, while ESS score > 10 indicates excessive daytime sleepiness.

Polysomnographic (PSG) assessment for objective sleep parameters by standard 8-hour overnight audio-videopolysomngraphy was performed in all subjects for one night using SOMNOscreen plus PSG+, 4238 apparatus, SOMNOmedics GmbH Am Sonnenstuhl 63; Germany. PSG evaluation included; an extended montage of electroencephalograms (EEG) (F3/A2, F4/A1, C3/A2, C4/A1, Cz/A1, O1/A2, O2/A1), electro-oculograms, submental electromyograms (EMGs) and bilateral anterior tibial EMGs for chin and tibialis anterior muscles, continuous electrocardiographic monitoring, nasal/oral airflow recorded with a thermistor, pulse oximetry and respiratory activity recorded with a thoracic and abdominal strain gauges (plethysmography). Subjects were under continuous videographic behavioral monitoring by a trained sleep technician in an adjacent room. After completion of the study, PSG scoring was done according to the American Academy of Sleep Medicine scoring rules; 2015 version 2.2 [16]. Rapid eye movement sleep behavior disorder was diagnosed according to the International Classification of Sleep Disorders-2 criteria. [17]. Apnea-hypopnea index (AHI) was calculated as the number of obstructive apneas, central apneas, or hypopneas per hour of sleep. Sleep disordered breathing (SDB) was diagnosed in patients with an AHI $\geq 5$ /hour. SDB was classified as mild (AHI 5-15/hour), moderate (AHI 15-30/hour), and severe (AHI $\geq 30$ /hour).

\section{Statistical Analysis:-}

Statistical package for social science (SPSS) (SPSS, Inc, Chicago, IL; Version 21) was used for data management and analysis. Kolmogorov-Smirnov test was done to test the normality of data distribution. Qualitative data were described using number and percentage. Chi square test was used to compare groups of categorical variables. Continuous variables were presented as mean \pm SD (standard deviation) for parametric data and median (range; minimum-maximum) for non-parametric data. Differences among nominal variables were analyzed using the X2 test or Fisher's exact test. General characteristics, clinical data of MS patients designated "good sleepers" (PSQI < 5) were compared with those for poor sleepers (PSQI > 5) using Student's t-test for normally distributed continuous variables; the Mann-Whitney U test was used for continuous variables that were not normally distributed. Student ttest and $\mathrm{x} 2$ test were used for the comparisons of all parameters between patients and controls. Correlations between global PSQI score and continuous variables were evaluated by means of Spearman correlation coefficients. Logistic regression was performed. Variables were included in the initial regression model if they were associated with sleep disorders with a $\mathrm{p}$ value $<0.10$. The results was considered significant when the probability of error is less than $5 \%$ $(\mathrm{p} \leq 0.05)$ at confidence interval $95 \%$.

\section{Results:-}

There was no significant difference in BMI or marital status between the patients and control groups. Demographic and clinical characteristics of the studied subjects are shown in (Table 1).

Table 1:- Demographic and clinical data of studied subjects.

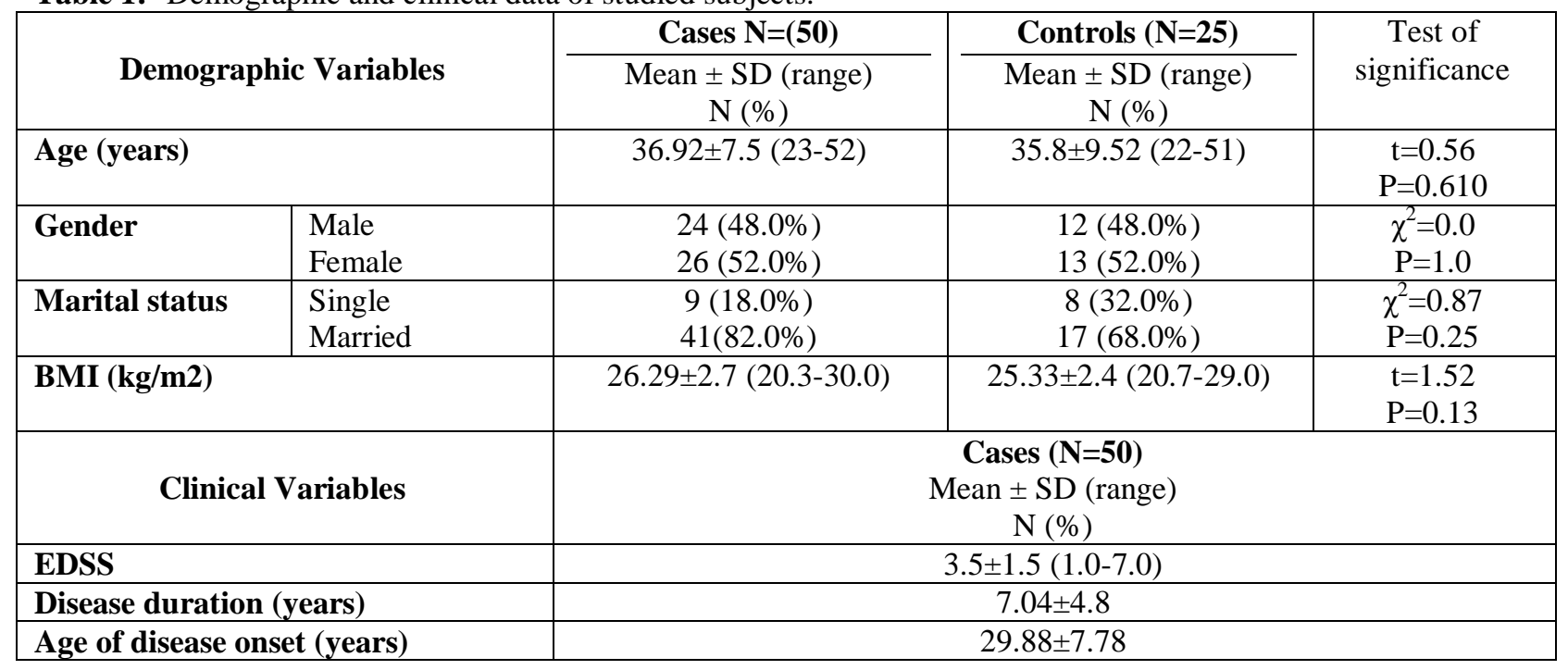




\begin{tabular}{|l|c|c|}
\hline Type & RRMS & $42(84.0 \%)$ \\
& SPMS & $8(16.0 \%)$ \\
\hline \multicolumn{2}{|l|}{ Number of attacks } & $5.4 \pm 3.1(2.0-15.0)$ \\
\hline RR (/year) & & $0.87 \pm 0.35(0.25-2.0)$ \\
\hline Medications & Pulse steroids & $12(24 \%)$ \\
& DMT (IFNb) & $12(24 \%)$ \\
\hline
\end{tabular}

$\chi^{2}=$ Chi-Square test $\quad t=$ Student $t$ test

Categorical variables expressed as numbers and proportions (\%). Continuous variables expressed as mean $\pm S D$ (standard deviation) and range. N= Number, BMI=Body Mass Index, EDSS=Extended Disability Status Scale, RR= Relapsing Rate, RRMS=Relapsing remittent multiple sclerosis, SPMS= secondary progressive multiple sclerosis, $D M T=$ Disease modifying therapy, IFN $b=$ interferon beta, $\mathrm{kg} / \mathrm{m} 2=$ kilogram per square meter

In this study, MS patients experience poor nocturnal sleep quality manifested by significant higher mean global scores on PSQI (8.48 \pm 3.4$)$ than controls $(2.44 \pm 1.4)$, with $82 \%$ of patients versus $4 \%$ of controls had poor sleep with subjective evidence of a sleep disturbance (PSQI >5), ( $\mathrm{p}<0.001)$. Compared with healthy controls, MS patients had statistically highly significant higher scores on ESS than controls, $(\mathrm{p}<0.001)$. Middle insomnia with difficult maintaining asleep and night awakening was the most common sleep complaint among MS patients (72\%), coming next to it, snoring (60\%), excessive daytime sleepiness (50\%), abnormal movements suggesting restless legs syndrome (RLS) (34\%), difficult breathing (32\%), while symptoms of abnormal behavior, with acting out dreams suggesting RBD was the least described in $8 \%$ of patients. All reported sleep complaints in MS patients were statistically significant higher compared to controls $(\mathrm{p}<0.05)$, apart from no statistical significant difference in the complaint of abnormal behavior with acting out dreams ( $\mathrm{p}=0.294)$. (Table 2).

Table 2:- Sleep problems among studied subjects.

\begin{tabular}{|c|c|c|c|c|}
\hline \multirow{2}{*}{\multicolumn{2}{|c|}{ Sleep variables }} & \multirow{3}{*}{$\begin{array}{c}\text { MS Cases }(\mathbf{N}=\mathbf{5 0}) \\
\text { Mean } \pm \text { SD (range) } \\
\text { N }(\%) \\
8.48 \pm 3.4(1-17) \\
9(18 \%) \\
41(82 \%)\end{array}$} & \multirow{3}{*}{$\begin{array}{c}\text { Controls }(\mathbf{N}=\mathbf{2 5}) \\
\text { Mean } \pm \text { SD (range) } \\
\text { N }(\%) \\
2.44 \pm 1.4(1-6) \\
24(96 \%) \\
1(4 \%)\end{array}$} & \multirow{3}{*}{$\begin{array}{c}\begin{array}{c}\text { Test of } \\
\text { significance }\end{array} \\
\\
\mathrm{t}=8.5 \\
\mathbf{P}<\mathbf{0 . 0 0 1 * *}\end{array}$} \\
\hline & & & & \\
\hline PSQI & $\begin{array}{l}\mathrm{PSQI} \leq 5 \\
\mathrm{PSQI}>5\end{array}$ & & & \\
\hline \multicolumn{2}{|l|}{ ESS } & $10.1 \pm 3.7(2-18)$ & $2.16 \pm 2.06(0-10)$ & $\begin{array}{c}\mathrm{t}=9.8 \\
\mathbf{P}<\mathbf{0 . 0 0 1} * *\end{array}$ \\
\hline \multicolumn{2}{|c|}{ Initial insomnia } & $23(46.0 \%)$ & $3(12.0 \%)$ & $\begin{array}{c}\chi^{2}=8.51 \\
\mathbf{P}=\mathbf{0 . 0 0 4} *\end{array}$ \\
\hline \multicolumn{2}{|c|}{ Middle insomnia } & $36(72.0 \%)$ & $0(0.0 \%)$ & $\begin{array}{c}\chi^{2}=34.6 \\
\mathbf{P}<\mathbf{0 . 0 0 1} * *\end{array}$ \\
\hline \multicolumn{2}{|c|}{ Excessive daytime sleepiness } & $25(50.0 \%)$ & $1(4.0 \%)$ & $\begin{array}{c}\chi^{2}=15.57 \\
\mathrm{P}<\mathbf{0 . 0 0 1} * *\end{array}$ \\
\hline \multicolumn{2}{|c|}{ Snoring } & $30(60.0 \%)$ & $8(32.0 \%)$ & $\begin{array}{c}\chi^{2}=5.23 \\
\mathbf{P}=\mathbf{0 . 0 2 2} *\end{array}$ \\
\hline \multicolumn{2}{|c|}{ Difficult breathing } & $16(32.0 \%)$ & $2(8.0 \%)$ & $\begin{array}{c}\text { FET } \\
\mathbf{P}=\mathbf{0 . 0 0 1} * *\end{array}$ \\
\hline \multicolumn{2}{|c|}{ Abnormal movement (RLS) } & $17(34.0 \%)$ & $2(8.0 \%)$ & $\begin{array}{c}\chi^{2}=5.96 \\
\mathrm{P}=\mathbf{0 . 0 1 5}\end{array}$ \\
\hline \multicolumn{2}{|c|}{ Abnormal behavior (RBD) } & $4(8.0 \%)$ & $0(0.0 \%)$ & $\begin{array}{c}\text { FET } \\
\mathrm{P}=0.294\end{array}$ \\
\hline
\end{tabular}

$t=$ Student $t$ test $\quad \chi^{2}=$ Chi-Square test $\quad F E T=$ Fischer exact test

Categorical variables expressed as numbers and proportions (\%). Continuous variables expressed as mean $\pm S D$

(standard deviation) and range.

$*$ = statistically significant $p$ value $\quad * *=$ highly statistically significant $p$ value

PSQI= Pittsburg Sleep Quality Index, ESS=Epworth Sleepiness Scale (ESS), RLS=Restless legs syndrome, RBD= REM behavior disorders

Two patients had no stage N3 sleep and ten patients had no REM sleep. Comparison of polysomnographic data between MS patients and the control group showed that, although, patient groups and controls did not differ 
significantly on REM latency and percentage of stage N3 or slow wave sleep (SWS), MS patients had significantly higher total arousal index (TAI), periodic limb movement index (PLMI), sleep onset latency (SOL), wake after sleep onset (WASO), stage shifts, percentage of stage N1 and percentage of stage N2, with lower sleep efficiency (SE), total sleep time (TST) and percentage of REM sleep than controls. Moreover, there was trend towards significant higher apnea hypopnea index (AHI) in patients than controls $(\mathrm{p}=0.069)$. (Table 3). RBD was confirmed by subsequent polysomnography with synchronized audio-visual recording with presence of REM without atonia (RWA) in only one patient (2\%). AHI $\geq 5$ suggesting sleep apnea syndrome was noted in 9 patients $(18 \%)$. Seven of these patients (14\%) had mild obstructive sleep apnea (OSA) with AHI between 5-15. One patient (2\%) had moderate OSA (AHI between 15 and 30) and one patient (2\%) had severe OSA (AHI >30). Ten patients (20\%) had periodic limb movement index (PLMI) $\geq 5$. Of these patients, seven (14\%) had mild periodic limb movement of sleep (PLMS) with a PLM index between 5-25, one (2\%) had moderate PLMS with an index between 25-50 and two (4\%) had severe PLMS with an index of $>50$.

Table 3:- Polysomnographic findings among studied subjects.

\begin{tabular}{|l|c|c|c|}
\hline \multicolumn{1}{|c|}{ Sleep parameters } & Cases & Controls & \multirow{2}{*}{$p$-value } \\
\cline { 2 - 4 } & $\begin{array}{c}\text { Median (min.-max.) } \\
\text { Mean } \pm \text { SD (range) }\end{array}$ & $\begin{array}{c}\text { Median (min.-max.) } \\
\text { Mean } \pm \text { SD (range) }\end{array}$ & \\
\hline Sleep efficiency (\%) & $72.4(43.50-97.20)$ & $90.5(74.40-97.90)$ & $\leq \mathbf{0 . 0 0 1 * *}$ \\
\hline Total sleep time (minutes) & $310.0(174.50-453.50)$ & $371.5(270.35-491.18)$ & $\leq \mathbf{0 . 0 0 1 * *}$ \\
\hline Sleep onset latency (minutes) & $20.93(2.35-100.11)$ & $11.46(1.81-25.46)$ & $\mathbf{0 . 0 0 2 *}$ \\
\hline REM latency (minutes) & $120.5(0.00-387.50)$ & $109.5(54.50-177.0)$ & 0.447 \\
\hline WASO (minutes) & $72.5(1.56-200.50)$ & $19.0(2.50-116.50)$ & $\leq \mathbf{0 . 0 0 1 * *}$ \\
\hline Stage shifts index (/hour) & $2.70(1.0-13.1)$ & $1.90(0.90-4.20)$ & $\mathbf{0 . 0 0 5} *$ \\
\hline \% stage N1 sleep & $12.25(3.60-33.80)$ & $6.20(1.60-10.20)$ & $\leq \mathbf{0 . 0 0 1} * *$ \\
\hline \% stage N2 sleep & $59.35(43.90-77.80)$ & $49.70(35.0-59.7)$ & $\leq \mathbf{0 . 0 0 1} * *$ \\
\hline \% stage N3 sleep & $21.9(0.00-43.10)$ & $22.10(16.20-37.10)$ & 0.278 \\
\hline \% REM sleep & $4.5(0.00-28.90)$ & $21.20(14.4-26.60)$ & $\leq \mathbf{0 . 0 0 1 * *}$ \\
\hline Total Arousal index & $14.68 \pm 11.2(2.70-68.60)$ & $2.86 \pm 1.9(0.00-6.10)$ & $\leq \mathbf{0 . 0 0 1} * *$ \\
\hline PLMI (/hour) & $6.01 \pm 18.4(0.00-92.0)$ & $0.09 \pm 0.3(0.00-1.30)$ & $\mathbf{0 . 0 0 5 *}$ \\
\hline AHI (/hour) & $3.04 \pm 7.4(0.00-44.40)$ & $0.48 \pm 0.8(0.00-3.40)$ & 0.069 \\
\hline
\end{tabular}

Continuous variables expressed as mean $\pm S D$ (standard deviation) for parametric and as median and range (min.max.) for non-parametric variables.

WASO = Wake after sleep onset, $P L M I=$ Periodic limb movements index, AHI=Apnea hypopnea index $*=$ statistically significant $p$ value $\quad * *=$ highly statistically significant $p$ value

In an univariate analysis, PSQI was significantly positive correlated with disability (EDSS) scores, disease duration, sleepiness (ESS), depression (BDI), anxiety (BAI), fatigue (FSS) scores and inversely correlated with quality of life (QoL). Moreover, PSQI was significantly associated with SPMS type, treatment with DMT, pulse steroids, pain, spasm and nocturia. However, PSQI scores were not correlated with age of disease onset, relapsing rate (RR) of the disease, demographic data and spasticity. (Table 4).

Comparing poor sleepers (PSQI $>5$ ) with good sleepers (PSQI $\leq 5$ ), EDSS score, prevalence of pain, spasm, nocturia, sleepiness (ESS), depression (BDI), anxiety (BAI), fatigue (FSS) due to MS, percentage of patients on DMT (IFB) and pulse intravenous steroids were significantly higher in poor sleepers than in good sleepers. However, there was no significant differences between each regards demographic, socioeconomic characteristics, other MS characters and spasticity. (Table 5).

Table 4:-Effects of different demographic, clinical variables and scales on sleep quality (PSQI).

\begin{tabular}{|l|c|c|}
\hline \multirow{2}{*}{ Variables } & \multicolumn{2}{c|}{ PSQI } \\
\cline { 2 - 3 } & Rho & $p$-value \\
\hline Beck depression scale & 0.670 & $<\mathbf{0 . 0 0 1} * *$ \\
\hline Beck anxiety scale & 0.673 & $<\mathbf{0 . 0 0 1} * *$ \\
\hline Fatigue severity scale & 0.602 & $<\mathbf{0 . 0 0 1} * *$ \\
\hline
\end{tabular}




\begin{tabular}{|c|c|c|c|}
\hline \multicolumn{2}{|l|}{ Quality of life index } & -0.677 & $<0.001 * *$ \\
\hline \multicolumn{2}{|l|}{ Epworth sleepiness scale } & 0.698 & $<0.001 * *$ \\
\hline \multicolumn{2}{|l|}{ EDSS } & 0.672 & $<0.001 * *$ \\
\hline \multicolumn{2}{|l|}{ Disease duration } & 0.321 & 0.023* \\
\hline \multicolumn{2}{|l|}{ Relapsing rate } & 0.086 & 0.553 \\
\hline \multicolumn{2}{|l|}{ Age of disease onset } & -0.057 & 0.692 \\
\hline \multicolumn{2}{|l|}{ Attacks number } & 0.406 & $0.003 *$ \\
\hline Type & $\begin{array}{l}\text { RRMS } \\
\text { SPMS }\end{array}$ & $\begin{array}{c}8.0(1.0-14.0) \\
10.0(8.0-17.0)\end{array}$ & 0.044* \\
\hline Disease modifying therapy & $\begin{array}{l}\text { Yes } \\
\text { No }\end{array}$ & $\begin{array}{l}9.5(8-17) \\
8.0(1-14)\end{array}$ & $0.025 *$ \\
\hline Pulse steroids & $\begin{array}{l}\text { Yes } \\
\text { No }\end{array}$ & $\begin{array}{l}11.0(1-17) \\
8.0(3-14)\end{array}$ & 0.034* \\
\hline \multicolumn{2}{|l|}{ Age } & 0.186 & 0.197 \\
\hline \multicolumn{2}{|l|}{ Body mass index } & 0.141 & 0.330 \\
\hline Gender & $\begin{array}{l}\text { Male } \\
\text { Female }\end{array}$ & $\begin{array}{l}8.00(1-14) \\
8.00(3-17)\end{array}$ & 0.945 \\
\hline Residence & $\begin{array}{l}\text { Rural } \\
\text { Urban }\end{array}$ & $\begin{array}{l}8.00(3-12) \\
8.00(1-17)\end{array}$ & 0.265 \\
\hline Educational level & $\begin{array}{l}\text { Low } \\
\text { High }\end{array}$ & $\begin{array}{l}9.00(1-14) \\
7.00(3-17)\end{array}$ & 0.312 \\
\hline Pain & $\begin{array}{l}\text { Yes } \\
\text { No }\end{array}$ & $\begin{array}{l}9.00(7-17) \\
6.00(1-17)\end{array}$ & $<0.0001 * *$ \\
\hline Spasm & $\begin{array}{l}\text { Yes } \\
\text { No }\end{array}$ & $\begin{array}{l}9.00(7-12) \\
8.00(1-17)\end{array}$ & 0.0494* \\
\hline Spasticity & $\begin{array}{l}\text { Yes } \\
\text { No }\end{array}$ & $\begin{array}{l}9.00(1-17) \\
6.00(3-14) \\
\end{array}$ & 0.063 \\
\hline Nocturia & $\begin{array}{l}\text { Yes } \\
\text { No }\end{array}$ & $\begin{array}{c}9.00(3-17) \\
6.00(1-9)\end{array}$ & $<0.0001 * *$ \\
\hline
\end{tabular}

Rho= Spearman's correlation coefficient, Mann-Whitney test

$*$ = statistically significant $\mathrm{p}$ value $\quad * *=$ highly statistically significant $\mathrm{p}$ value

PSQI= Pittsburg Sleep Quality Index, EDSS= Extended Disability Status Scale

Table 5:-Different demographic, clinical variables and scales in patients with poor and good slee

\begin{tabular}{|c|c|c|c|c|}
\hline \multicolumn{2}{|c|}{ Variables } & $\begin{array}{c}\text { Good sleepers }(\mathbf{N}=\mathbf{9}) \\
\text { Mean } \pm \text { SD } \\
\text { Median (Min. - Max.) } \\
\text { N (\%) }\end{array}$ & $\begin{array}{c}\text { Poor sleepers }(\mathbf{N}=\mathbf{4 1}) \\
\text { Mean } \pm \text { SD } \\
\text { Median (Min. - Max.) } \\
\text { N (\%) }\end{array}$ & $\mathrm{P}$ value \\
\hline \multicolumn{2}{|l|}{ Age (years) } & $35.11 \pm 4.22$ & $37.32 \pm 7.79$ & 0.427 \\
\hline \multicolumn{2}{|l|}{ BMI $(\mathrm{kg} / \mathrm{m} 2)$} & $24.90(22.7-29.5)$ & $27.00(20.3-30.0)$ & \\
\hline Gender & \begin{tabular}{|l} 
Male \\
Female
\end{tabular} & $\begin{array}{l}4(16.7 \%) \\
5(19.2 \%)\end{array}$ & $\begin{array}{l}20(83.3 \%) \\
21(80.8 \%)\end{array}$ & 1.00 \\
\hline Residence & $\begin{array}{l}\text { Rural } \\
\text { Urban }\end{array}$ & $\begin{array}{l}6(24.0 \%) \\
3(12.0 \%)\end{array}$ & $\begin{array}{l}19(76.0 \%) \\
22(88.0 \%)\end{array}$ & 0.463 \\
\hline Educational level & $\begin{array}{l}\text { Low } \\
\text { High }\end{array}$ & $\begin{array}{l}4(12.1 \%) \\
5(29.4 \%)\end{array}$ & $\begin{array}{l}29(87.9 \%) \\
12(70.6 \%)\end{array}$ & 0.242 \\
\hline Marital status & \begin{tabular}{|l|} 
Single \\
Married
\end{tabular} & $\begin{array}{l}1(11.1 \%) \\
8(19.5 \%)\end{array}$ & $\begin{array}{c}8(88.9 \%) \\
33(80.5 \%)\end{array}$ & 1.00 \\
\hline \multicolumn{2}{|c|}{ Age of disease onset (years) } & $31.00(23-38)$ & $29.0(18-46)$ & 0.781 \\
\hline \multicolumn{2}{|c|}{ Disease duration (years) } & $4.00(3-12)$ & $6.0(2-25)$ & 0.230 \\
\hline Number of attack & & $4.00(2-6)$ & $5.0(2-15)$ & \\
\hline
\end{tabular}




\begin{tabular}{|c|c|c|c|c|}
\hline & & & & 0.229 \\
\hline Relapsing rate & & $1.00(0.25-2.00)$ & $0.83(0.25-2.00)$ & 0.721 \\
\hline EDSS & & $2.05 \pm 1.01$ & $3.81 \pm 1.46$ & \\
\hline Tyne & RRMS & $9(214 \%)$ & $33(786 \%)$ & \\
\hline & SPMS & $0(0.0 \%)$ & $8(100.0 \%)$ & 0.322 \\
\hline Epworth sleep & scale & $5.0(2-12)$ & $11.0(6-18)$ & \\
\hline & & & & $0.001 * *$ \\
\hline Beck depressi & & $11.56 \pm 2.87$ & $18.95 \pm 5.08$ & \\
\hline & & & & $<0.001 * *$ \\
\hline Beck Anxiety & & $12.0(9-15)$ & $21.00(9-33)$ & $<0.001 * *$ \\
\hline Fatigue severi & & $21.0(16-49)$ & $40.0(21-56)$ & $0.001 * *$ \\
\hline Quality of life & & $84.00(67-95)$ & $59.00(23-83)$ & \\
\hline Pulse steroid & \begin{tabular}{|l} 
No \\
Yes
\end{tabular} & $\begin{array}{c}18(47.4 \%) \\
1(8.3 \%)\end{array}$ & $\begin{array}{l}20(52.6 \%) \\
11(91.7 \%)\end{array}$ & $0.042 *$ \\
\hline DMT & $\begin{array}{l}\text { No } \\
\text { Yes }\end{array}$ & $\begin{array}{l}19(50 \%) \\
0(0.0 \%)\end{array}$ & $\begin{array}{c}19(50 \%) \\
12(100.0 \%)\end{array}$ & $0.05 *$ \\
\hline Pain & $\begin{array}{l}\text { Absent } \\
\text { Present }\end{array}$ & $\begin{array}{c}9(39.1 \%) \\
0(0.0 \%)\end{array}$ & $\begin{array}{c}14(60.9 \%) \\
27(100.0 \%)\end{array}$ & $<0.001 * *$ \\
\hline Spasm & $\begin{array}{l}\text { Absent } \\
\text { Present }\end{array}$ & $\begin{array}{c}25(55.5 \%) \\
0(0.0 \%) \\
\end{array}$ & $\begin{array}{l}20(44.5 \%) \\
5(100.0 \%)\end{array}$ & $0.047 *$ \\
\hline Spasticity & $\begin{array}{l}\text { Absent } \\
\text { Present }\end{array}$ & $\begin{array}{l}7(17.1 \%) \\
2(22.2 \%)\end{array}$ & $\begin{array}{c}34(82.9 \%) \\
7(77.8 \%)\end{array}$ & 0.657 \\
\hline Nocturia & $\begin{array}{l}\text { Absent } \\
\text { Present }\end{array}$ & $\begin{array}{c}7(41.2 \%) \\
2(6.1 \%)\end{array}$ & $\begin{array}{l}10(58.8 \%) \\
31(93.9 \%)\end{array}$ & $0.004 *$ \\
\hline
\end{tabular}

$*=$ statistically significant $\mathrm{p}$ value $\quad * *=$ highly statistically significant $\mathrm{p}$ value

EDSS=Extended Disability Status Scale, BMI=Body Mass Index, DMT=Disease modifying therapy, RRMS = Relapsing remittent $M S, \quad S P M S=$ Secondary progressive $M S$

In multivariate analysis, depression, anxiety, pain, nocturia, fatigue, DMT (immunotherapy), high disability (EDSS), poor quality of life (QoL) were considered as independent predictors for poor sleep. (Table 6).

Table 6:-Logistic regression analysis for prediction of poor sleep (PSQI >5).

\begin{tabular}{|l|c|c|}
\hline \multirow{2}{*}{ Variables } & Poor sleep (PSQI > 5) \\
\cline { 2 - 3 } & OR $(95 \%$ CI) & P value \\
\hline Beck depression inventory & $7.968(0.835-12.063)$ & $\mathbf{0 . 0 4 1}^{*}$ \\
\hline Beck anxiety inventory & $5.309(0.428-.955)$ & $\mathbf{0 . 0 3 6}^{*}$ \\
\hline Fatigue severity scale & $8.991(0.823-30.281)$ & $\mathbf{0 . 0 2 5}^{*}$ \\
\hline Quality of life index & $7.864(2.754-19.562)$. & 0.0637 \\
\hline Epworth sleepiness scale & $3.073(0.190-8.051)$ & 0.190 \\
\hline Disease duration & $1.523(0.812-2.856)$ & 0.073 \\
\hline Number of attacks & $0.460(0.197-1.074)$ & $\mathbf{0 . 0 2 6}$ \\
\hline EDSS & $3.309(0.428-6.755)$ & 0.066 \\
\hline MS type & $0.015(0.001-3.607)$ & 0.981 \\
\hline Pulse steroid & $1.036(0.054-19.826)$ & $\mathbf{0 . 0 3 2}^{*}$ \\
\hline Disease modifying therapy & $5.638(1.54-13.785)$ & $\mathbf{0 . 0 2 1 6}^{*}$ \\
\hline Pain & $7.921(0.601-14.43)$ & 0.758 \\
\hline Spasm & $1.572(0.088-28.08)$ & $\mathbf{0 . 0 0 6}^{*}$ \\
\hline Nocturia & $24.92(2.532-45.44)$ & \\
\hline
\end{tabular}

* = statistically significant $\mathrm{p}$ value 
$\mathrm{OR}=$ Odds Ratio, $C I=$ Confidence interval, PSQI= Pittsburg Sleep Quality Index, EDSS $=$ Extended Disability Status Scale

\section{Discussion:-}

Our study shows that, $82 \%$ of our patients reported poor sleep based on PSQI. Unlike demographic and socioeconomic variables, clinical conditions, both related and unrelated to MS, are associated with an altered quality of sleep in individuals affected by MS. Moreover, our study underscores and confirms important points that sleep disorders are common in MS patients though often unrecognized and also, sleep disorders are often associated with disability, pain, bladder dysfunction, fatigue and mood disorders leading to impairment of QoL.

In agreement with our study, several studies [18-22] showed that in MS patient group, there was higher number of nocturnal awakenings, stage shifts index and WASO, with lower SE compared with controls. Other studies [23, 24] found increased sleep stage changes and shifts in the subjects with sleep disorders compared to those without and in MS patients than matched controls. Moreover, in concordance with our study, some researchers found that MS patients had significant longer sleep latency, WASO and higher number of awakenings with less SE and TST compared to the controls [25, 26]. Furthermore, two studies [27, 28] found that MS patients had significantly reduced SE \% and increased sleep latency, compared to control group.

However, in contrast to our study, SE and sleep latency did not differ significantly between MS patients and healthy controls in some previous studies [5, 23, 24, 29]. Moreover, another study [30] found that patient groups and controls did not differ significantly on TST, SL, SE, WASO percentage and stage shifts. The reasons of this difference may be that Kaminska and colleagues included patients with relapse-free for at least 30 days prior to screening and during the study, and no chronic steroid treatment for $\geq 6$ months prior to study entry. Lunde and colleagues depended on self-report questionnaires but not on objective measures as PSG or actigraphy like our study. In addition, authors of another study reported that the difference in TST between women with and without MS was not significant $(p=0.16)[31]$.

The present study showed a significant increase in the median TAI and PLMI in MS cases than controls, reflecting disruption of sleep microstructure, in agreement with several previous studies $[\mathbf{1 8}, \mathbf{2 0 - 2 2 , ~ 2 4 , ~ 2 8 , 3 0 ] . ~}$

The current study showed significant increased stage N1 and N2 NREM sleep percentage (light sleep) in MS patients in comparison to controls $(\mathrm{p}<0.001)$, in agreement with some studies [21, 22]. Moreover, authors of another study [24] found increased N1 sleep in the sleep disorder (SD)-subjects compared with the no SD subjects. We reported absent stage N3 sleep in two patients (4\%), with no significant difference between MS patients and normal controls $(\mathrm{p}=0.278)$, in agreement with several studies $[\mathbf{1 8}, \mathbf{2 3}, \mathbf{2 4}, \mathbf{2 9}, \mathbf{3 0}, \mathbf{3 2}$. We reported absent stage REM sleep in 13 patients (26\%), with median REM sleep percentage was reduced in MS patients compared to normal controls $(\mathrm{p} \leq 0.001)$, in agreement with some two previous studies $[\mathbf{2 2}, \mathbf{2 8}]$.

Moreover, in the present study, there was a trend towards significant difference between MS patients and controls regards AHI ( $\mathrm{p}=0.069)$, in agreement with findings of another study [23]. In addition, authors of other two studies $[29,30]$ found no significant differences in AHI between patient group and controls.

Ninety six percentage of MS patients were reported to have objective evidence of relevant sleep disturbances in a previous study [33]. Moreover, authors in another study [23] reported as high as $80 \%$ of MS patients had sleep disorders when describing incidence of OSA in a clinical sample. In addition, some researchers found that poor sleep was present in $77 \%$ of patients [29]. Other authors in their study [34] found that $80 \%$ of patients reported sleep problems. Furthermore, $85 \%$ of patients in another study [35] endorsed at least one nocturnal symptom to interfere with their ability to get a good night's sleep. More than $87 \%$ of MS patients of another study experienced sleep problems [36]. In another study [37] authors found that $93.75 \%$ of MS patients had objective evidence of a sleep disturbance. These previous studies are consistent with and near similar to our study, that $82 \%$ of our MS patients had poor sleep.

With respect to subjective sleep data, we reported a significant higher mean global PSQI score compared with the healthy controls, in agreement with different previous studies $[5,20,30,38-43]$. In the current study, there was no significant association between demographic characteristics (gender or age) and poor sleep, in agreement with two previous studies $[4,44]$. 
In the current study, there was a significant positive correlation between PSQI and EDSS ( $p<0.001$ ), disease duration ( $\mathrm{p}=0.023)$, with significant higher EDSS and percentage of SPMS in poor than good sleepers. It could be assumed that increased disability would lead to increased sleep impairment due to restricted movement with difficulty in turning in or getting out of bed unaided, decreased bladder control, progressive neurological symptoms and increased psychological strain. Some studies $[4,30,38,39,45-48]$ suggested that higher EDSS and longer MS duration are associated with a greater prevalence of sleep disorders and higher PSQI, in agreement with our study.

In the present study, there was significant association between poor sleep and usage of immunotherapy (DMT, IFNb), with higher percentage of poor sleepers taking DMT than good sleepers, in agreement with some previous studies $[\mathbf{5}, \mathbf{4 4}, \mathbf{4 9}, \mathbf{5 0}]$; however, in contrast to other studies $[4, \mathbf{4 5}, \mathbf{4 6}, \mathbf{5 1}]$.

In our study, depression, anxiety, pain, nocturia, fatigue, DMT (immunotherapy), high disability (EDSS) and poor quality of life (QoL) were considered as independent predictors for poor sleep in MS patients in linear regression modeling. In agreement with our study, depression, pain, fatigue and nocturia were found to be associated with reduced quality of sleep in one previous study [52]. In an another study [5], authors found that poor sleep was independently associated with use of immunotherapy, reduced quality of life and the high psychological burden of MS. Moreover, in a multivariate analysis of another study [45], their authors reported depression, disability, nocturia, pain and fatigue were associated with poor sleep. In addition, two more studies [48, 53] found that multivariate logistic regression analysis indicated that patients with higher fatigue were more likely to have poor sleep quality.

Moreover, in concordance with our study, some authors in their study [54] found that, in a linear regression model variables statistically significantly related with sleep problems included higher level of disability (EDSS), fatigue, pain, bladder/sexual dysfunction, depression and anxiety. No relations were found between independent variables like residence, education, marital status, disease duration and sleep problems. In addition, a significant regression coefficient between sleep quality and disability, depression, pain and physical fatigue was reported in another study [55]. Furthermore, multivariate logistical regression analysis of another study [43] revealed that higher psychological burden of MS and depression might be risk factors for poor sleep in MS patients.

In the current study; ESS score was significantly higher in MS patients than in controls, $(\mathrm{p}<0.001)$. Fifty percentage of MS patients reported excessive daytime sleepiness (EDS) versus none of the controls $(\mathrm{p}<0.001)$, in concordance with previous studies $[21,29,31,37, \mathbf{4 4}, \mathbf{4 5}, \mathbf{4 8}-\mathbf{5 1}, \mathbf{5 7}, \mathbf{5 8}]$. In agreement with our study, several studies $[\mathbf{3 1}, \mathbf{4 4}$, $\mathbf{4 5}, \mathbf{5 6}]$ found that ESS scores were positively correlated with disturbed sleep.

\section{Conclusion:-}

Sleep disorders are an underestimated problem in MS patients. We concluded that sleep disturbances are very frequent in MS patients and more prevalent than general population, with poor sleep quality was reported in $82 \%$ of our patients; middle insomnia in 72\%; initial insomnia in 46\%; EDS in 50\%; RLS in 34\%; PLMD in 20\%; sleep apnea in $18 \%$; while RBD was only reported in $2 \%$ of patients with MS. There are several variables contributing to disturbed sleep in our MS patients. Different factors of depression, anxiety, pain, nocturia, fatigue, DMT (immunotherapy), high disability (EDSS) and poor quality of life (QoL) were considered as independent predictors for poor sleep in MS patients. 


\section{References:-}

1. Čarnická Z, Kollár B, Šiarnik P, Krížová L, Klobučníková K, Turčáni P. Sleep disorders in patients with multiple sclerosis. J Clin Sleep Med 2015;11:553-557.

2. Ferini-Strambi L. Sleep disorders in multiple sclerosis. In: Michael JA, Francois B, Dick FS, Montagna P, Chokroverty S, editors. Handbook of clinical neurology. Third edition. Amsterdam: Elsevier; 2011. p. $1139-1146$.

3. Caminero A, Bartolomé M. Sleep disturbances in multiple sclerosis. J Neurol Sci 2011;309:86-91.

4. Merlino G, Fratticci L, Lenchig C, Valente M, Cargnelutti D, Picello M, et al. Prevalence of 'poor sleep'among patients with multiple sclerosis: an independent predictor of mental and physical status. Sleep Med 2009;10:26-34.

5. Lunde HMB, Aae TF, Indrevåg W, Aarseth J, Bjorvatn B, Myhr K-M, et al. Poor sleep in patients with multiple sclerosis. PloS one. 2012;7:e49996.

6. Fleming WE, Pollak CP. Sleep disorders in multiple sclerosis. Semin Neurol 2005;25:64-68.

7. Polman CH, Reingold SC, Banwell B, Clanet M, Cohen JA, Filippi M, et al. Diagnostic criteria for multiple sclerosis: 2010 revisions to the McDonald criteria. Ann Neurol 2011;69:292-302.

8. Kurtzke JF. Rating neurologic impairment in multiple sclerosis: an expanded disability status scale (EDSS). Neurology 1983;33:1444-52.

9. Folstein MF, Folstein SE, McHugh PR. "Mini-mental state": A practical method for grading the cognitive state of patients for the clinician. J Psychiatr Res 1975;12:189-98.

10. Buysse DJ, Reynolds CF, Monk TH, Berman SR, Kupfer DJ. The Pittsburgh Sleep Quality Index: a new instrument for psychiatric practice and research. Psychiatry Res 1989;28:193-213.

11. Johns MW. A new method for measuring daytime sleepiness: the Epworth sleepiness scale. Sleep 1991;14:540-5.

12. Krupp LB, LaRocca NG, Muir-Nash J, Steinberg AD. The fatigue severity scale: application to patients with multiple sclerosis and systemic lupus erythematosus. Arch Neurol 1989;46:1121-3.

13. Beck A, Ward C, Mendelson M, Mock J, Erbaugh J. An inventory for measuring depression. Arch Gen Psychiatry 1961;4:561-71.

14. Beck AT, Epstein N, Brown G, Steer RA. An inventory for measuring clinical anxiety: psychometric properties. J Consult Clin Psychol 1988; 56:893-897.

15. Stiasny-Kolster K, Mayer G, Schäfer S, Möller JC, Heinzel-Gutenbrunner M, Oertel WH. The REM sleep behavior disorder screening questionnaire - a new diagnostic instrument. Mov Disord 2007;22:2386-93.

16. Berry RB, Brooks R, Gamaldo CE, Harding SM, Marcus C, Vaughn B. for the American Academy of Sleep Medicine. The AASM manual for the scoring of sleep and associated events. Rules, Terminology and Technical Specifications, version 2.2. Darien, Illinois, USA: American Academy of Sleep Medicine; 2015.

17. American Academy of Sleep Medicine. In: Winkleman J, Kotagal S, Olson E, Scammel T, Schenk C, editors. International classification of sleep disorders: diagnostic and coding manual. 2nd ed. Westchester, IL (US): American Academy of Sleep Medicine. 2005; 51-55.

18. Ferini-Strambi L, Filippi M, Martinelli V, Oldani A, Rovaris M, Zucconi M, et al. Nocturnal sleep study in multiple sclerosis: correlations with clinical and brain magnetic resonance imaging findings. J Neurol Sci 1994;125:194-7.

19. Antonijevic IA, Steiger A. Depression-like changes of the sleep-EEG during high dose corticosteroid treatment in patients with multiple sclerosis. Psychoneuroendocrinology 2003;28:780-95.

20. Kaynak H, Altintaş A, Kaynak D, Uyanik Ö, Saip S, Ağaoğlu J, et al. Fatigue and sleep disturbance in multiple sclerosis. Eur J Neurol 2006;13:1333-9.

21. Kaminska M, Kimoff R, Schwartzman K, Trojan D. Sleep disorders and fatigue in multiple sclerosis: evidence for association and interaction. J Neurol Sci 2011;302:7-13.

22. Trojan DA, Kaminska M, Bar-Or A, Benedetti A, Lapierre Y, Da Costa D, et al. Polysomnographic measures of disturbed sleep are associated with reduced quality of life in multiple sclerosis. J Neurol Sci 2012;316:158-63.

23. Braley TJ, Segal BM, Chervin RD. Sleep-disordered breathing in multiple sclerosis. Neurology 2012;79:92936.

24. Côté I, Trojan D, Kaminska M, Cardoso M, Benedetti A, Weiss D, et al. Impact of sleep disorder treatment on fatigue in multiple sclerosis. Mult Scler 2013;19:480-9.

25. Elkattan MM, El-Serafy OA, Helmy SM, Kishk NA, Shaheen HA, Belal ES, et al. Exploring the relationship between fatigue and sleep disturbances in multiple sclerosis. Egypt J Neurol Psychiat Neurosurg 2009;46:193-202. 
26. Mendozzi L, Tronci F, Garegnani M, Pugnetti L. Sleep disturbance and fatigue in mild relapsing remitting multiple sclerosis patients on chronic immunomodulant therapy: an actigraphic study. Mult Scler 2010;16:23847.

27. Melamud L, Golan D, Luboshitzky R, Lavi I, Miller A. Melatonin dysregulation, sleep disturbances and fatigue in multiple sclerosis. J Neurol Sci 2012;314:37-40.

28. Elnabil L, Ibrahim E. Polysomnographic picture of multiple sclerosis: Correlation with MRI findings. Sleep Med 2013;14:e115.

29. Kaminska M, Kimoff R, Benedetti A, Robinson A, Bar-Or A, Lapierre Y, et al. Obstructive sleep apnea is associated with fatigue in multiple sclerosis. Mult Scler 2012;18:1159-69.

30. Chen J-H, Liu X-Q, Sun H-Y, Huang Y. Sleep disorders in multiple sclerosis in China: clinical, polysomnography study, and review of the literature. J Clin Neurophysiol 2014;31:375-81.

31. Li Y, Munger K, Batool-Anwar S, De Vito K, Ascherio A, Gao X. Association of multiple sclerosis with restless legs syndrome and other sleep disorders in women. Neurology 2012;78:1500-6.

32. Vetrugno R, Stecchi S, Scandellari C, Pierangeli G, Sabattini L, D'Angelo R, et al. Sleep-wake and body core temperature rhythms in multiple sclerosis with fatigue. Clin Neurophysiol 2007;118:228-34.

33. Veauthier C, Radbruch H, Gaede G, Pfueller C, Dörr J, Bellmann-Strobl J, et al. Fatigue in multiple sclerosis is closely related to sleep disorders: a polysomnographic cross-sectional study. Mult Scler 2011;17:613-22.

34. Kiziria M, Chikadze A, Khuchua L, Jibladze M, Tsiskaridze A, Shakarishvili R. Multiple sclerosis associated fatigue and sleep disturbances. Sleep Med 2013;14:e169.

35. Braley TJ, Segal BM, Chervin RD. Obstructive sleep apnea and fatigue in patients with multiple sclerosis. J Clin Sleep Med 2014;10:155-162.

36. Razazian N, Najafi F, Mahdavi P, Aghaei A. Prevalence of sleep disorders in patients with multiple sclerosis. J Mazandaran Univ Med Sci 2014;23:219-24.

37. Sater R, Gudesblatt M, Kresa-Reahl K, Brandes D, Sater P. The relationship between objective parameters of sleep and measures of fatigue, depression, and cognition in multiple sclerosis. Mult Scler J Exp Transl Clin 2015;1:2055217315577828.

38. Lobentanz I, Asenbaum S, Vass K, Sauter C, Klösch G, Kollegger H, et al. Factors influencing quality of life in multiple sclerosis patients: disability, depressive mood, fatigue and sleep quality. Acta Neurol Scand 2004;110:6-13.

39. Bamer AM, Johnson K, Amtmann D, Kraft G. Prevalence of sleep problems in individuals with multiple sclerosis. Mult Scler 2008;14:1127-30.

40. Ghajarzadeh M, Sahraian MA, Fateh R, Daneshmand A. Fatigue, depression and sleep disturbances in Iranian patients with multiple sclerosis. Acta Med Iran 2012;50:244-249.

41. Jalilian R, Ghajarzadeh M, Fateh R, Togha M, Sahraian MA, Azimi A. Comparison of Sleep Quality in Women with Migraine Moreover, Multiple Sclerosis. Acta Med Iran 2014;52:690-693.

42. Ahmadi F, Alamdary MP, Abedini M. Sleep quality among multiple sclerosis patients: A cross sectional study. Mintage Journal of Pharmaceutical and Medical Sciences 2017;6:26-27.

43. Ma S, Rui X, Qi P, Liu G, Yang J. Sleep disorders in patients with multiple sclerosis in China. Sleep Breath 2017;21:149-54.

44. Pokryszko-Dragan A, Bilińska M, Gruszka E, Biel $\mathbf{L}$, Kamińska K, Konieczna K. Sleep disturbances in patients with multiple sclerosis. J Neurol Sci 2013;34:1291-6.

45. Neau J-P, Paquereau J, Auche V, Mathis S, Godeneche G, Ciron J, et al. Sleep disorders and multiple sclerosis: a clinical and polysomnography study. Eur Neurol 2012;68:8-15.

46. Leonavicius R, Adomaitiene V. Features of sleep disturbances in multiple sclerosis patients. Psychiatr Danub 2014;26:249-255.

47. Vitkova M, Gdovinova Z, Rosenberger J, Szilasiova J, Nagyová I, Mikula P, et al. Factors associated with poor sleep quality in patients with multiple sclerosis differ by disease duration. Disabil Health J 2014;7:466-71.

48. Nociti V, Losavio FA, Gnoni V, Losurdo A, Testani E, Vollono C, et al. Sleep and fatigue in multiple sclerosis: A questionnaire-based, cross-sectional, cohort study. J Neurol Sci 2017;372:387-92.

49. Brass SD, Duquette P, Proulx-Therrien J, Auerbach S. Sleep disorders in patients with multiple sclerosis. Sleep Med Rev 2010;14:121-9.

50. Nadjar Y, Coutelas E, Prouteau P, Panzer F, Paquet D, Saint-Val C, et al. Injection of interferon-beta in the morning decreases flu-like syndrome in many patients with multiple sclerosis. Clin Neurol Neurosurg 2011;113:316-22. 
51. Labuz-Roszak B, Kubicka-Bączyk K, Pierzchała K, Machowska-Majchrzak A, Skrzypek M. Fatigue and its association with sleep disorders, depressive symptoms and anxiety in patients with multiple sclerosis. Neurol Neurochir Pol 2012;46:309-17.

52. Bamer AM, Johnson KL, Amtmann DA, Kraft GH. Beyond fatigue: Assessing variables associated with sleep problems and use of sleep medications in multiple sclerosis. Clin Epidemiol 2010;2:99-106.

53. Kara B, Tenekeci EG, Demirkaya S. Factors associated with sleep quality in patients with multiple sclerosis. AIMS Medical Science 2016;3:203-12.

54. Leonavicius R. Peculiarities of sleep problems in multiple sclerosis. Neurol Psychiat BR 2015;21:148-52.

55. Vitkova M, Rosenberger J, Gdovinova Z, Szilasiova J, Mikula P, Groothoff JW, et al. Poor sleep quality in patients with multiple sclerosis: gender differences. Brain Behav 2016;6:e00553.

56. Popp RF, Fierlbeck AK, Knüttel H, König N, Rupprecht R, Weissert R, et al. Daytime sleepiness versus fatigue in patients with multiple sclerosis: a systematic review on the Epworth sleepiness scale as an assessment tool. Sleep Med Rev 2017;32:95-108.

57. Attarian HP, Brown KM, Duntley SP, Carter JD, Cross AH. The relationship of sleep disturbances and fatigue in multiple sclerosis. Arch Neurol 2004;61:525-8.

58. Stanton B, Barnes F, Silber E. Sleep and fatigue in multiple sclerosis. Mult Scler 2006;12:481-6. 